\section{Economics and Value of IS}

\author{
Kunsoo Han • Dennis Kundisch • Christof Weinhardt • \\ Steffen Zimmermann
}

Published online: 28 July 2015

(C) Springer Fachmedien Wiesbaden 2015

\section{Introduction}

After the first and the second industrial revolution (textile industry enabled by water- and steam-powered mechanical manufacturing facilities in the 18th century, and mass production enabled by electric power in the early 20th century), a third, digital revolution is under way, radically transforming not just business but also social life (Economist 2012). In the digital transformation age, information technologies (IT) and information systems (IS) are the key drivers behind 'disruptive innovations', so called because new digital technologies enable new business models to emerge which create new markets and value networks and thereby disrupt existing markets by displacing an earlier technology (cf., Manyika et al. 2013). A prime example of disruptive innovation is online social networking which has

Assoc. Prof. K. Han

Information Systems, Desautels Faculty of Management, McGill

University, Montreal, QC, Canada

e-mail: kunsoo.han@mcgill.ca

Prof. Dr. D. Kundisch $(\bowtie)$

Chair of Information Management and E-Finance, Faculty of

Business Administration and Economics, University of

Paderborn, 33095 Paderborn, Germany

e-mail: dennis.kundisch@wiwi.uni-paderborn.de

Prof. Dr. C. Weinhardt

Institute of Information Systems and Marketing (IISM),

Information and Market Engineering (IM), Karlsruhe Institute of

Technology, 76131 Karlsruhe, Germany

e-mail: weinhardt@kit.edu

Assoz. Prof. Dr. S. Zimmermann

Information Systems II, School of Management, University

Innsbruck, 6020 Innsbruck, Austria

e-mail: Steffen.Zimmermann@uibk.ac.at changed the way people communicate and connect with each other. Another, even more radical and much debated, potential disruptive innovation is collaborative consumption, also known as the sharing of durable goods (Matzler et al. 2015), which is predicted to replace ownership. Both these phenomena rest on new business models that were made possible by Internet-based IS (e.g., facebook.com as online social networking platform and airbnb.com as online sharing platform) and Web 2.0 as a new version of the world wide web allowing users to interact and collaborate with each other. Other debated disruptive technologies that may have far-reaching consequences for consumers, firms, policy makers and societies as a whole include, for instance, big data technologies, mobile Internet, Internet of Things (including wearables), cloud technologies, and 3D printing (see, e.g., Manyika et al. 2013).

For firms and policy makers alike it is very important to know which of the upcoming technologies have the potential to result in disruptive innovations, and how these technologies may impact existing business models and markets and create value for society. Accordingly, there is a key role for researchers in the age of digital transformation, first, to provide information by analyzing and predicting the economic effects of disruptive IT, and second, by designing new IS, new business models, and new markets that are enabled by these technologies.

Such undertakings require an in-depth understanding of the underlying strategic challenges faced by each of the different players in the new markets (e.g., consumers, firms, policy makers), and of the various behavioral patterns of organizations and human subjects. It is hardly surprising, then, that disciplines that provide valuable guidelines for analyzing the economic effects of disruptive IS and market design, notably economic theory, experimental economics, and behavioral economics, have 
received increasing attention in recent years. Game-theory and experimental economics, for instance, have informed the design of auctions, two-sided markets, reputation mechanisms, etc. Economic theory, crucially, provides the basis for the design of policy measures to mitigate negative externalities that are created by disruptive IS such as online sharing platforms. Microeconomic theory also contributes valuable models for the equilibrium behavior of players in markets and other complex strategic environments. However, the design of disruptive IS poses many new challenges. For example, IS designers often face utility functions and design desiderata that are quite different from those described in microeconomic textbooks. Moreover, disruptive IS require the implementation and analysis of completely new business models and markets, which have not yet been described in the literature.

The extant literature in economic theory has prioritized the development of formal models that explain economic interactions, rather than systems design. On the other hand, the traditional design science literature on information systems, computer science, and operations research rarely consider incentives or the strategic interactions of individuals. In recent years there has been an increasing interaction between these different academic disciplines (e.g., algorithmic game theory) to their mutual benefit. A central theme in the IS literature has always been the interaction of information systems design and human behavior. Examples include the literature on the adoption of IS in organizations, but also the research on recommender systems where algorithm design and behavioral models need to be considered together. Therefore, it is not surprising that an increasing number of scholars focus on economic principles of IS design. Over time, and alongside this development, many invaluable forums have emerged where IS issues are discussed through the lens of economics, further underscoring the relevance for this type of research. These forums include: the Workshop on Information Systems and Economics (WISE), the Workshop on Theory in Economics of Information Systems (TEIS) (with a special focus on analytical modeling), Statistical Challenges in eCommerce Research (SCECR) (with a special focus on quantitative empirical work/big data), the 'Economics and Value of IS'-Tracks at the European Conference on Information Systems (ECIS), and the International Conference on Information Systems (ICIS), to name but a few.

While the topic 'Business value of IS/IT' has a strong design-oriented history in BISE and its predecessor WIRTSCHAFTSINFORMATIK (see, e.g., Wehrmann et al. 2006; Walter and Spitta 2004; Urbach et al. 2009), the topic 'Economics of IS/IT' was just recently introduced into BISE in 2012 with the re-organization of the departmental structure of the journal. We welcome this decision as BISE with its design-oriented tradition might be able to establish an engineering arm of economics, being rooted in economic theory.

\section{Papers in this Special Issue}

We received eleven papers for the special issue, and after initial screening, all eleven manuscripts were sent for review. We finally accepted three papers after up to three rounds of reviews, which yields an acceptance rate of below $30 \%$. This shows that associate editors and reviewers worked hard to maintain and demand consistent quality, which is evident in the papers that made it through the review process. What is noteworthy about the selected papers is that each addresses an area of current and at the same time prolonged research interest, IT decision making, sharing, and net neutrality - each making use of a different method.

The first paper, "The business value of IT in light of prospect theory - a new explanation for IT paradoxes" by Afflerbach (FIM Research Center, Germany) addresses a key problem with IT decision-making. The actual value contributions of IT projects are unknown ex-ante to their executions and, thus, an organization has to rely on the expectations and perceptions of its decision makers, which are prone to biases. The author analyzes how these biases are related to the business value of IT and how IT decisionmaking can be improved by using an analytical decision model based on prospect theory. The author proposes that the irrationalities rooted in human value perception provide explanations for two central paradoxes of IT, namely the "productivity paradox of IT" and the "perception paradox of IT".

The second paper, "The economics of multi-hop ride sharing: creating new mobility networks through IS", by Teubner and Flath (Karlsruhe Institute of Technology, Germany, and University of Würzburg, Germany) addresses sharing as a potential disruptive innovation by studying the idea of multi-hop ride sharing and illustrating how IS can leverage its potential. Based on empirical ride sharing data, the authors provide a quantitative analysis of the structure and the economics of electronic ride sharing markets, explore the potential and competitiveness of multi-hop ride sharing, and analyze its implications for platform operators. They find that multi-hop ride sharing proves competitive compared to other modes of transportation and has the potential to greatly increase ride availability and city connectedness, especially under high reliability requirements. To fully realize this potential, platform operators should implement, amongst others, multi-hop search, improve coordination of transfers, and enhance data services. 
The third paper, "When 'just' is just not enough: why consumers do not appreciate non-neutral internet access services" by Krämer and Wiewiorra (University of Passau, Germany, and Goethe University Frankfurt, Germany), deals with the issue of restricting net neutrality regulation that would substantially change the character of the current Internet technology. The authors argue that ISPs are reluctant to tap the price discrimination potential of prioritized Internet access services, because in the context of the ongoing public debate on net neutrality, such differentiation would be considered as unfair by their customers. Empirically analyzing a dataset of German Internet access customers, the authors find that the customers' perceptions of justice as well as the framing of the mechanism by which prioritized Internet access is provided are indeed decisive for whether customers prefer this access regime over net neutrality. Interestingly, the authors find that customers are more likely to accept a regime that offers an absolute rather than a relative prioritization of data flows.
Acknowledgments We thank all the authors of the submitted papers, the associate editors, and the reviewers for their contributions, and the managing editor and editorial office for their guidance and assistance in the preparation process.

\section{References}

Economist (2012) The third industrial revolution. http://www. economist.com/node/21553017. Accessed 1 Jul 2015

Manyika J, Chui M, Bughin J, Dobbs R, Bisson P, Marrs A (2013) Disruptive technologies: advances that will transform life, business, and the global economy. McKinsey Global Institute, USA

Matzler K, Veider V, Kathan W (2015) Adapting to the sharing economy. MIT Sloan Manag Rev 56(2):70-77

Urbach N, Smolnik S, Riempp G (2009) The state of research on information systems success - a review of existing multidimensional approaches. Bus Inf Syst Eng 1(4):315-325

Walter SG, Spitta T (2004) Approaches to the ex-ante evaluation of investments into information systems. Wirtschaftsinformatik 46(3):171-180

Wehrmann A, Heinrich B, Seifert F (2006) Quantitative IT portfolio management towards a value based approach to risk management of IT investments. Wirtschaftsinformatik 48(4):234-245 